\title{
Assessment of tissue ischemia of nail fold precapillary zones using a fluorescence capillaroscopy
}

Viktor V. Dremin, Nikita B. Margaryants, Mikhail V. Volkov, Ekaterina V. Zhukova, Evgeny A. Zherebtsov, et al.

Viktor V. Dremin, Nikita B. Margaryants, Mikhail V. Volkov, Ekaterina V. Zhukova, Evgeny A. Zherebtsov, Andrey V. Dunaev, Edik U. Rafailov, "Assessment of tissue ischemia of nail fold precapillary zones using a fluorescence capillaroscopy," Proc. SPIE 10412, Diffuse Optical Spectroscopy and Imaging VI, 104120W (28 July 2017); doi: 10.1117/12.2284223

SPIE. Event: European Conferences on Biomedical Optics, 2017, Munich, Germany 


\title{
Assessment of Tissue Ischemia of Nail Fold Precapillary Zones Using a Fluorescence Capillaroscopy
}

\author{
Viktor V. Dremin*a , Nikita B. Margaryants ${ }^{\mathrm{b}}$, Mikhail V. Volkov ${ }^{\mathrm{b}}$, Ekaterina V. Zhukova ${ }^{\mathrm{b}}$, \\ Evgeny A. Zherebtsov ${ }^{\mathrm{a}, \mathrm{c}}$, Andrey V. Dunaev ${ }^{\mathrm{a}}$, Edik U. Rafailov ${ }^{\mathrm{c}}$ \\ ascientific-Educational Center "Biomedical Engineering", \\ Orel State University named after I.S. Turgenev, Orel, 302026, Russia; \\ bITMO University, Saint-Petersburg, 197101, Russia \\ 'Optoelectronics and Biomedical Photonics Group, Aston Institute of Photonic Technologies, \\ Aston University, Aston Triangle, Birmingham, B4 7ET, UK
}

\begin{abstract}
An optical instrument for nailfold fluorescence capillaroscopy and image registration has been developed. With this instrument, an effect of increasing fluorescence intensity in the spectral range of NADH fluorescence during ischemia was detected.
\end{abstract}

Keywords: fluorescence, capillaroscopy, imaging, metabolism.

\section{INTRODUCTION}

Currently, pre-clinical and clinical studies show that the use of diagnostic methods based on detection of fluorescence radiation, can improve the early diagnosis of a cancer and other destructive processes in various organs ${ }^{1}$. New designs of instruments and analytical tools for obtaining new diagnostic information are currently intensively developing. However, instruments still require a significant improvement for their large-scale introduction into clinical practice.

Many factors which have a strong impact on the results of fluorescence measurements should be considered. In case of in vivo and in situ measurements, new approaches of analysis and processing of obtained data are required to get reliable diagnostical information. So, fluorescence spectroscopy measurements of the two places of tissue with the same distribution and concentration of fluorophores, but with a different blood volume, will provide significantly different spectra due to the strong impact of blood on parameters of light absorption in spectral range of visible light. Without appropriate data correction of the measurements, the accurate determination of fluorophore concentration and even their ratio cannot be achieved ${ }^{2-4}$. For the successful applications of the methods of fluorescence spectroscopy in clinical practice it is important to find out reliable, but easy to use method of the correction.

To solve the task in this study, we have simultaneously applied the measurements of several parameters of biological tissue. Fluorescence and diffuse reflectance imaging techniques ${ }^{5,6}$, as well as the method of videocapillaroscopy ${ }^{7,8}$ during local occlusion tests were chosen for the approach. Areas of fingers' proximal nail fold was chosen for the investigations. This area is easily available for visual control by the videocapillaroscopy method to quantitatively assess the parameters of blood flow as well as fluorescence intensity of adjoining precapillary zones. The areas are of particular interest to in vivo tissue gaseous exchange investigation. Such measurements provide comprehensive diagnostic information with the possibility of correction of the recorded fluorescence data. It makes it possible to study the effects of delivery and consumption of oxygen in vivo and in situ, as well as the impact of blood flow on metabolism of living biological tissues.

Thus, the aim of the study was investigation of respiratory chain parameters by recording NADH fluorescence intensity of nail fold epithelial tissues supplemented by simultaneous tissue reflectance measurements during local tissue ischemia.

*dremin_viktor@mail.ru; phone 74862 419876; bmecenter.ru/en

Diffuse Optical Spectroscopy and Imaging VI, edited by Hamid Dehghani, Heidrun Wabnitz,

Proc. of SPIE-OSA Vol. 10412, 104120W · c 2017 OSA-SPIE

CCC code: $1605-7422 / 17 / \$ 18 \cdot$ doi: $10.1117 / 12.2284223$ 


\section{MATERIAL AND METHODS}

In this study, the area of the proximal nail fold of the middle finger was illuminated by LED light source with a $365 \mathrm{~nm}$ central wavelength (power $\sim 2 \mathrm{~mW}$ ) and a broadband halogen source HL-2000 (Ocean Optics, USA, 360-1000 nm, $\sim 7 \mathrm{~mW}$ ). A high-aperture microscope objective with a numerical aperture of 0.12 and a projection long-focus objective were used to form the scaled-up image on a monochrome CCD sensor. Filtering of fluorescent images was carried out by using bandpass optical filters (Fig.1).

To induce tissue ischemia and dynamically monitor the changes of fluorescence intensity, the occlusion tests was conducted (by imposition of the cuff on the brachial artery). Thus, the artificial state of ischemia was created. NADH molecules formed in the sixth glycolysis reaction, depending on the presence of oxygen, have two paths of further transformation. They can either stay in the cytosol and enter the eleventh reaction of glycolysis (anaerobic conditions), or to penetrate the mitochondria and become oxidised in the respiratory chain of the Krebs cycle (aerobic conditions). In the conditions of hypoxia, mitochondrial NADH oxidation is slowed down. Also in the case of the glycolysis (anaerobic) path, NADH formation is activated. In this regard, it can be assumed that the detected level of fluorescence during this experiment should increase ${ }^{9}$.

In the protocol of the study, occlusion tests with pressure of $220 \mathrm{mmHg}$ during $1.5 \mathrm{~min}$ were conducted. A pair of images (fluorescence and reflectance) were recorded before and at the end of the occlusion test. At the next stage, image drift stabilization of the study area and frame alignment were conducted.

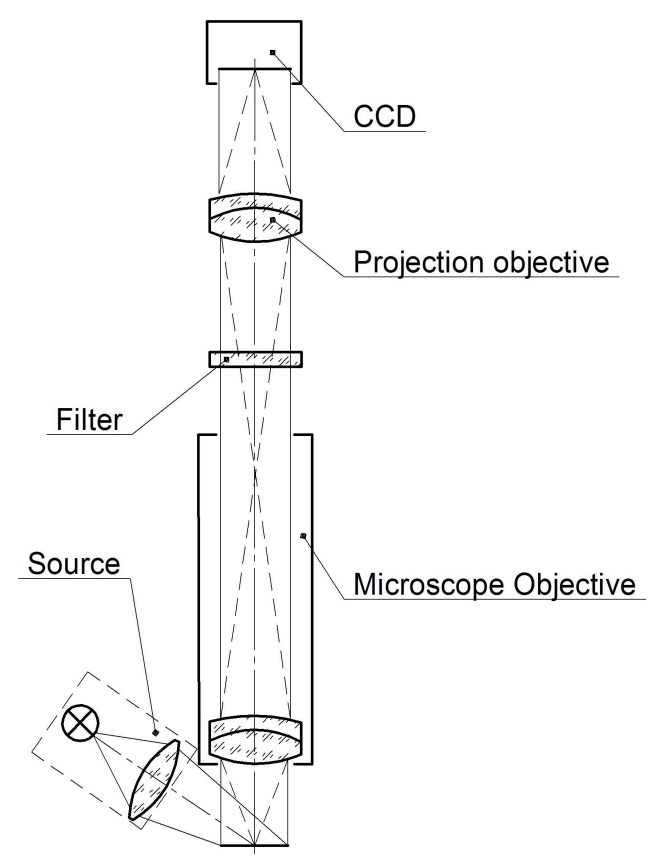

Figure 1. The experimental setup.

Reflectance images were calculated as follows below:

$$
I_{d}(\lambda)=\frac{I_{t}(\lambda)-I_{b}(\lambda)}{I_{P T F E}(\lambda)-I_{b}(\lambda)}
$$

where $I_{t}(\lambda)$ - the measured reflectance image of biological tissue; $I_{P T F E}(\lambda)$ - the measured reflectance image of the etalon optical diffuser (PTFE); $I_{b}(\lambda)$ - the background image obtained without any illumination. 


\section{EXPERIMENTAL RESULTS AND DISCUSSION}

Obtained fluorescence images of precapillary zones normalised to the image of the diffuse reflection from experimental studies are presented in Fig. 2.

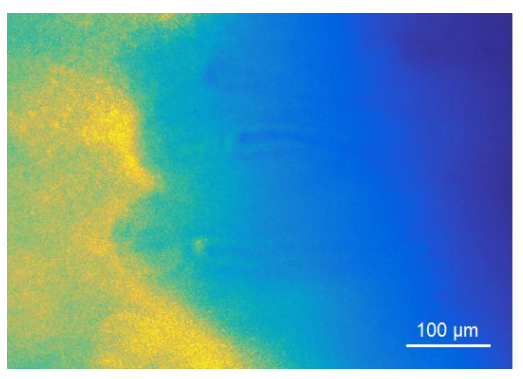

a)

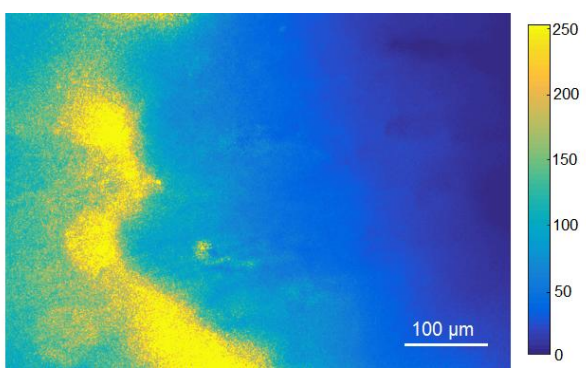

b)

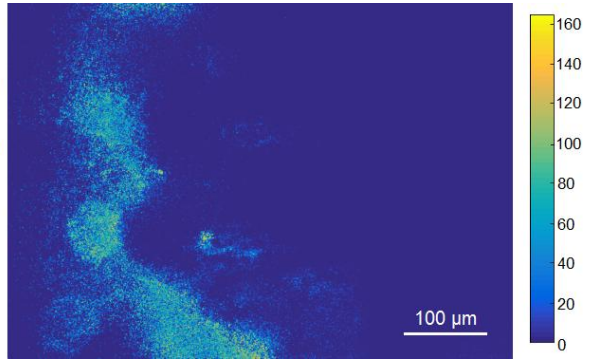

c)

Figure 2. Fluorescence images before (a), in the end (b) of occlusion test and difference between stages (c)

It can be seen that by the end of the occlusion test, the fluorescence intensity significantly increases. The effect is especially bright in the precapillary zones, which may indicate a significant accumulation of NADH due to tissue hypoxia.

Thus, the proposed approach can be used to study the dynamics of changes and NADH in vivo and in situ. In vivo registration of NADH fluorescence as a noninvasive marker for the detection of cell death is promising for the development of highly efficient ways to detect and treat cancer ${ }^{10-12}$.

\section{CONCLUSION}

These promising and substantial results demonstrate the ability of spectroscopic techniques to provide useful information for disease classification in a noninvasive manner. Specifically, fluorescence changes of NADH will provide details about tissue biochemistry.

The conducted study can be of particular interest in the research area of cell metabolism as well as find applications in clinical practice. However, further research is needed to understand the reliable diagnostic criteria based on imaging of NADH fluorescence in epithelial tissues.

\section{ACKNOWLEDGMENTS}

The work was supported by grant of the President of the Russian Federation for state support of young Russian scientists № MK-7168.2016.8 and H2020 MCSA funded project, grant number 703145.

\section{REFERENCES}

[1] Mycek, M. A.., Pogue, B. W., Handbook of Biomedical Fluorescence, Taylor \& Francis (2003).

[2] Dremin, V. V., Dunaev, A. V., "How the melanin concentration in the skin affects the fluorescence-spectroscopy signal formation,” J. Opt. Technol. 83(1), 43-48 (2016).

[3] Dremin, V. V., Zherebtsov, E. A., Rafailov, I. E., Vinokurov, A. Y., Novikova, I. N., Zherebtsova, A. I., 
[3] Dremin, V. V., Zherebtsov, E. A., Rafailov, I. E., Vinokurov, A. Y., Novikova, I. N., Zherebtsova, A. I., Litvinova, K. S.., Dunaev, A. V., "The development of attenuation compensation models of fluorescence spectroscopy signals," Proc. SPIE 9917, 99170Y (2016).

[4] Dunaev, A. V., Dremin, V. V., Zherebtsov, E. A., Rafailov, I. E., Litvinova, K. S., Palmer, S. G., Stewart, N. A., Sokolovski, S. G.., Rafailov, E. U., "Individual variability analysis of fluorescence parameters measured in skin with different levels of nutritive blood flow," Med Eng Phys 37(6), 574-583 (2015).

[5] Leblond, F., Davis, S. C., Valdes, P. A., Pogue, B. W., "Pre-clinical whole-body fluorescence imaging: Review of instruments, methods and applications," J. Photochem. Photobiol. B. 98(1), 77-94 (2010).

[6] Bish, S. F., Sharma, M., Wang, Y., Triesault, N. J., Reichenberg, J. S., Zhang, J. X. J.., Tunnell, J. W., "Handheld Diffuse Reflectance Spectral Imaging (DRSi) for in-vivo characterization of skin," Biomed. Opt. Express 5(2), 573-586 (2014).

[7] Cutolo, M., Pizzorni, C., Sulli, A., “Capillaroscopy,” Best Pract. Res. Clin. Rheumatol. 19(3), 437-452 (2005).

[8] Volkov, M. V., Kostrova, D. A., Margaryants, N. B., Gurov, I. P., Erofeev, N. P., Dremin, V. V., Zharkikh, E. V., Zherebtsov, E. A., Kozlov, I. O., Dunaev, A. V., "Evaluation of blood microcirculation parameters by combined use of laser Doppler flowmetry and videocapillaroscopy methods," Proc. SPIE 10336, 1033607 (2017).

[9] Mayevsky, A., Chance, B., "Oxidation-reduction states of NADH in vivo: from animals to clinical use," Mitochondrion 7(5), 330-339 (2007).

[10] Rafailov, I., Palmer, S., Litvinova, K., Dremin, V., Dunaev, A., Nabi, G., "A novel excitation-emission wavelength model to facilitate the diagnosis of urinary bladder diseases," Proc. SPIE 9303, 93030W (2015).

[11] Rafailov, I. E., Dremin, V. V., Litvinova, K. S., Dunaev, A. V., Sokolovski, S. G.., Rafailov, E. U., "Computational model of bladder tissue based on its measured optical properties," J. Biomed. Opt. 21(2), 025006 (2016).

[12] Drezek, R., Sokolov, K., Utzinger, U., Boiko, I., Malpica, A., Follen, M., Richards-Kortum, R., “Understanding the contributions of NADH and collagen to cervical tissue fluorescence spectra: modeling, measurements, and implications," J. Biomed. Opt. 6(4), 385-396 (2001). 